www.nature.com/clinicalpractice/uro

\title{
Living beyond cancer: survivorship is more than surviving
}

Mary S McCabe

Owing to the recent advances in diagnosis and treatment, the number of cancer survivors is currently estimated to be 22.4 million individuals worldwide (President's Cancer Panel [2004] Living Beyond Cancer: A European Dialogue. Bethesda, MD: National Cancer Institute, $\mathrm{NIH}$ ). According to the SEER database, the 5-year relative survival rate for adults diagnosed in the mid-1990s is approximately $64 \%$, and exceeds $90 \%$ for individuals diagnosed with earlystage prostate cancer (www.seer.cancer.gov); however, along with these optimistic results come new challenges for the cancer survivor, including the need for ongoing medical care and psychosocial services. This is particularly true for the survivors of urologic cancers who, as a consequence of treatment, might experience physical problems that affect sexual intimacy and self image, which have a tremendous effect on quality of life. We now know that survivors face a variety of ongoing health risks that are dependent on a number of factors: the type of cancer, treatment exposures, genetic predisposition, comorbid health conditions and lifestyle behaviors. So how should we care for this large number of individuals? Where do we start?

Just as the pediatric oncology community established follow-up clinics in which consensus-based guidelines form the basis for survivorship care, the adult oncology community should now make the post-treatment period a distinct phase of care as well. Currently, adult follow-up care is variable in duration, tends to focus on cancer recurrence, and, in busy practices, survivors are often lost to follow-up. Thus, it is essential to develop a comprehensive, clinical visit tailored to the specific needs of survivors. We have a rapidly growing body of knowledge about the long-term and late effects of cancer therapy that is sufficient to use and evaluate, even as we continue to research who

\section{Shared care \\ between \\ providers \\ has become \\ standard for \\ the successful \\ management \\ of chronic \\ diseases... and \\ is applicable \\ to the care \\ of the cancer \\ survivor...}

MS McCabe is

Director of the Cancer

Survivorship Program

at Memorial Sloan-

Kettering Cancer Center, New York, NY, USA.

Competing interests

The author declared no

competing interests.

www.nature.com/clinicalpractice doi:10.1038/ncpuro0947 is at risk and for what problems. At a minimum, the survivorship visit should include surveillance for recurrence, evaluation and treatment of medical and psychosocial consequences of treatment, recommendations for screening for new primary cancers, health promotion recommendations such as diet, exercise and smoking cessation, and provision of a written care plan to the patient and primary care physician. These essential care components can be provided in an efficient manner regardless of whether the survivor is being followed in a cancer center, community hospital or physician office.

In addition to developing a systematic approach to follow-up, communication between the oncology specialist and other physician groups who provide care needs to improve. Since survivors, who might face lifetime risks of future health problems, receive care for comorbid diseases from their primary care (family) physician, it is critical to develop and evaluate a model of 'shared care' between the oncology specialist and these generalists. Shared care between providers has become standard for the successful management of chronic diseases, such as diabetes and chronic renal disease, and is applicable to the care of the cancer survivor as well. It involves a periodic transfer of knowledge about potential risks and ongoing communication about health status. For survivors at low risk of recurrence and late effects of treatment, a transition to the primary care physician might also be appropriate.

Despite health systems barriers and an evolving knowledge base, we need to begin to evaluate new, innovative models of follow-up care, participate in guideline development, and support survivorship research that will provide the evidentiary base upon which to develop programs and services. Only then will we truly move beyond survival to survivorship care. 